\title{
Organic Semiconductor-Polymer Insulator Blends: a Morphological Study of the Guest-Host Interactions*
}

\author{
Marie-Beatrice Madec, ${ }^{\dagger}$ David J. Crouch, and Gonzalo Rincon-LLorente \\ OMIC, School of Chemistry, University of Manchester, Oxford Road, Manchester M13 9PL, UK \\ Tracie J. Whittle \\ Department of Chemistry, Richard Roberts Building, \\ University of Sheffield, Brook Hill, Sheffield S3 $7 H F$, UK \\ Mark Geoghegan \\ Department of Physics and Astronomy, Hicks Building, \\ University of Sheffield, Hounsfield Road, Sheffield S3 $7 R H$, UK \\ Stephen G. Yeates \\ OMIC, School of Chemistry, University of Manchester, Oxford Road, Manchester M13 9PL, UK.
}

(Received 1 June 2008; Accepted 8 October 2008; Published 4 April 2009)

\begin{abstract}
We suggest a link between film morphology and electrical properties of a crystalline organic semiconductor: polymer insulating blend. TIPS-Pentacene (6,13-bis(triisopropylsilylethynyl) pentacene) [J. Am. Chem. Soc. 123, 9482 (2001)] is formulated with various amorphous and semi-crystalline side chain aromatic low permittivity dielectric polymers. Film structure investigated by AFM microscopy and XPS gives invaluable information regarding film morphology and confirms potential intermolecular interaction. All these morphological observations were linked to the electrical characterisation of organic thin film transistors (OTFTs) and the determination of saturated hole mobility value. When an amorphous binder is used the more dilute the TIPS-pentacene the lower the saturated hole mobility value. When a semi-crystalline binder is used it is possible to massively dilute TIPS-pentacene within the polymer dielectric without loosing either the crystalline structure of the material or the electronic properties. Besides phase segregation, enrichment of the active interface in TIPS-Pentacene is demonstrated.
\end{abstract}

[DOI: 10.1380/ejssnt.2009.455]

Keywords: Atomic force microscopy; X-Ray photoelectron spectroscopy; Surface structure, morphology, roughness, topography; Crystalline-amorphous interfaces

\section{INTRODUCTION}

During the last decade, organic thin film transistors (OTFTs) have become increasingly more attractive for various electronic applications. Notably, devices based on organic semiconductors have the advantages of potentially low cost solution processability, and for migration to flexible substrates. However to date OTFTs cannot achieve the high switching frequency of single crystalline silicon because of the inherently lower mobility and structural disorder of organic compounds in the solid state. This is further compounded by issues of reliability and reproducibility when deposited over large areas particularly when the organic semiconductor is a crystalline material. For that reason, we have focused our effort on formulation of crystalline organic semiconductor - low permittivity $(\varepsilon)$ polymer dielectric nanocomposites with a view to improving film quality and reproducibility over large areas. Ultimately, our goal is to understand the effects of films morphology on device performance with a view to developing robust solution processable formulations.

OTFT's based on pentacene have exhibited to date the best performance with saturated field effect mobilities up to $3 \mathrm{~cm}^{2} \mathrm{~V}^{-1} \mathrm{~s}^{-1}$ for vacuum deposited and $35 \mathrm{~cm}^{2} \mathrm{~V}^{-1}$

${ }^{*}$ This paper was presented at the 14th International Conference on Solid Films and Surfaces (ICSFS-14), Trinity College Dublin, Ireland, 29 June - 4 July, 2008.

†Corresponding author: marie-beatrice.madec@manchester.ac.uk
TABLE I: Physical properties of low permittivity insulator polymer binders.

\begin{tabular}{ccccc}
\hline \hline Binder & Mn (daltons) & $T_{\mathrm{g}}\left({ }^{\circ} \mathrm{C}\right)$ & $T_{\mathrm{m}}\left({ }^{\circ} \mathrm{C}\right)$ & $\varepsilon$ at $1 \mathrm{KHz}^{(a)}$ \\
\hline PAMS & 1300 & 74 & - & 2.6 \\
PS & 350,000 & 100 & - & 2.5 \\
iPS & 400,000 & 100 & 212 & 2.6 \\
iPVN & 100,000 & 162 & 360 & 2.6 \\
\hline \hline
\end{tabular}

${ }^{(a)}$ low frequency permittivity

$\mathrm{s}^{-1}$ for single crystal devices, respectively [1]. In our work a functionalized pentacene derivative, TIPS-pentacene $(6$, 13-bis(triisopropylsilylethynyl) pentacene) has been prepared and when formulated with various amorphous and semi-crystalline low $\varepsilon$ polymers dielectric binders a range of composites have been evaluated as organic field effect transistor.

\section{EXPERIMENTAL}

1,2,3,4-tetrahydronaphthalene (tetralin, anhydrous 99\%), $\quad \operatorname{poly}(\alpha$-methylstyrene) (PAMS), amorphous polystyrene (PS), semi-crystalline isotactic polystyrene (iPS), semi-crystalline isotactic polyvinylnaphthalene (iPVN), and octadecyl trichlorosilane (OTS) were all obtained from Sigma-Aldrich UK and used as received. 


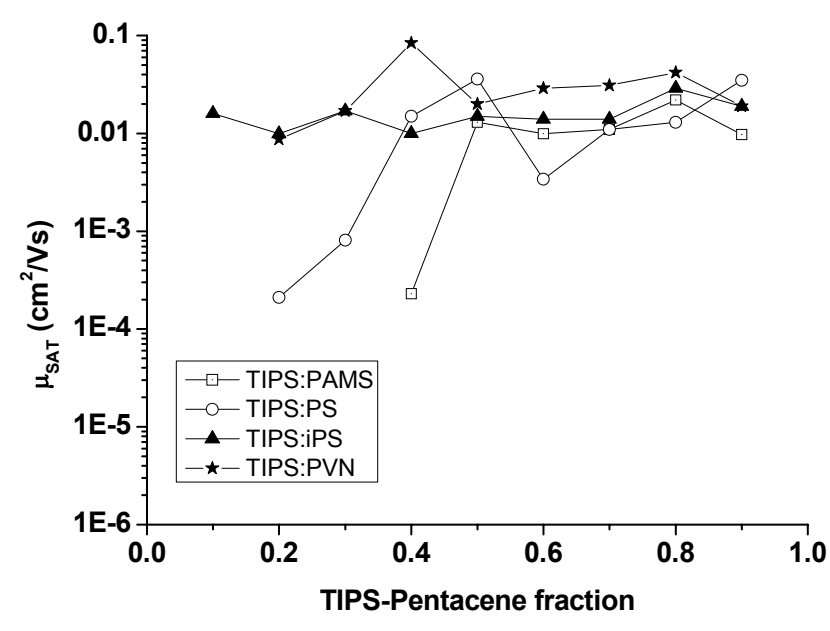

FIG. 1: Saturated hole mobility of TIPS-Pentacene-insulator blends as a function of weight fraction of TIPS-pentacene for PAMS, PS, and iPS.

6,13-bis(triisopropylsilylethinyl)pentacene (TIPS-pentacene), melting point $=261^{\circ} \mathrm{C}$, was synthesized according to the method previously described by Anthony [2]. Physical properties of various binders are stated in Table I. Tetralin was chosen for the study as solubility studies show it to be a good solvent for all components and its high boiling point $\left(210^{\circ} \mathrm{C}\right)$ makes it suitable for future micro-deposition studies. Blends were prepared fresh prior to making the devices. The total mass content was kept constant at $1 \% \mathrm{w} / \mathrm{w}$, with the fraction of TIPS-pentacene being varied from 0.1 to 0.9 .

Discrete bottom-gate, top-contact field effect transistor (FET) devices were fabricated on OTS treated heavily n-doped silicon wafers comprising a $3000 \AA$ thermally grown gate oxide layer. Substrates were cleaned by sonication in de-ionised water for 15 minutes, then acetone for 15 minutes and finally methanol for 15 minutes, prior to immersion in a solution of $10 \mathrm{mM}$ OTS in toluene for 20 minutes maintained at $60^{\circ} \mathrm{C}$. Substrates were then dried on a hot plate for 2 hours at $150^{\circ} \mathrm{C}$ under normal atmosphere. $1 \mathrm{wt}-\%$ tetralin solution of various TIPSpentacene-insulator blends were deposited and dried at room temperature under vacuum $(0.1 \mathrm{mBar} / 60$ minutes $)$ to remove any entrained solvent. No post thermal annealing was employed. Gold source and drain channel electrodes, $60 \mu \mathrm{m}$ length and $2 \mathrm{~mm}$ width were thermally evaporated, at $10^{-6} \mathrm{mBar}$ until a layer thickness of $50 \mathrm{~nm}$ was obtained, yielding 9 transistors per substrate.

Devices were characterized in DC current using an E5270B 8Slot precision Measurement frame from Agilent Technology coupled to a 3 Agilent E5287A atto level High Resolution Module. Contacts were made using Karl Süss PH100 manual microprobes. To remain in the saturated regime, the value of the drain voltage was kept equal to the gate voltage at all times. From the slope of the square root of the drain intensity characteristic, we were able to calculate the saturated hole mobility $\left(\mu_{\mathrm{FET}}\right)$ and the threshold voltage.

Scanning Force Microscopy (SFM) was performed on an AFM XE100 (PSIA Inc, Seoul, Korea) in contact mode at $1 \mathrm{~Hz}$ and a constant contact force of $21.4 \mathrm{nN}$. We used
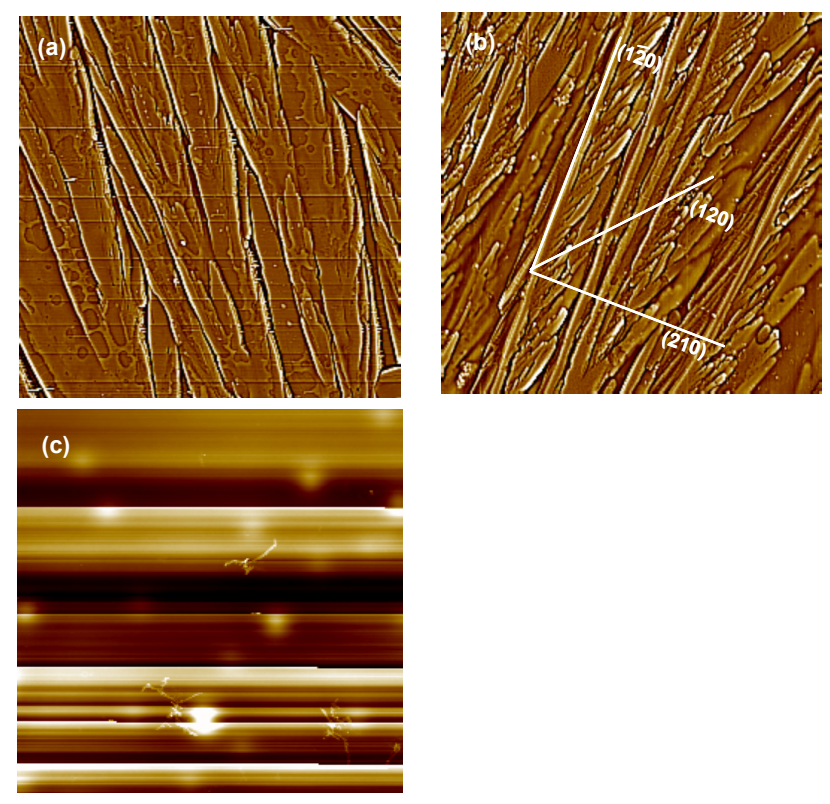

FIG. 2: SFM Topographic images of TIPS-Pentacene: iPS for (a) (90:10) formulation, (b) (10:90) formulation and (c) TIPSPentacene: PAMS (10:90) formulation. (Contrast enhanced AFM contact mode image, $50 \mu \times 50 \mu \mathrm{m}, 1 \mathrm{~Hz}$.)

a soft commercial silicon cantilever with a nominal spring constant of about $0.6 \mathrm{~N} / \mathrm{m}$, the back of the cantilever being coated with aluminum to increase signal feedback.

X-Ray Photoelectron spectroscopy (XPS) was measured using a Kratos Axis Spectrometer. A monochromated $\mathrm{Al} \mathrm{K} \alpha$ at a power of $150 \mathrm{~W}$ was used to acquire the spectra under secondary vacuum of $10^{-6} \mathrm{~Pa}$. For each sample, measurements were recorded for top interface and bottom interface (by lifting off the film from the silicon wafer).

\section{RESULTS AND DISCUSSIONS}

Figure 1 shows the evolution of the saturated hole mobility $\left(\mu_{\text {sat }}\right)$ as a function of weight fraction of TIPSpentacene when it is formulated with each binder. We observe that for all blends having a TIPS-pentacene weight fraction $\geq 0.5$ the saturated hole mobility is $\geq$ $0.01 \mathrm{~cm}^{2} /$ Vs which is one order better than for pure TIPSpentacene under the same conditions. This improvement may be attributed either to a self-encapsulation [3] effect where the binder protects the active semiconductor from oxidative environment or to improved contact due to the physical properties of the polymer: organic crystal solution. At weight fractions $<0.5$ TIPS-pentacene the saturated hole mobility and is strongly dependent upon the nature of the polymeric binder falling off rapidly when it is amorphous, $<10^{-6} \mathrm{~cm}^{2} /$ Vs at 0.2 in PAMS, but being retained at weight fraction of TIPS-pentacene $\leq 0.2$ when it is crystalline.

All devices show differing levels of anticlockwise hysteresis in the $I_{\mathrm{d}} / V_{\mathrm{g}}$ curves which is reproducible upon cycling of the device, which maybe attributed to intrinsic dynamic doping of the TIPS-pentacene $[4,5]$. Upon decreasing the weight fraction of TIPS-pentacene hysteresis 
(a)

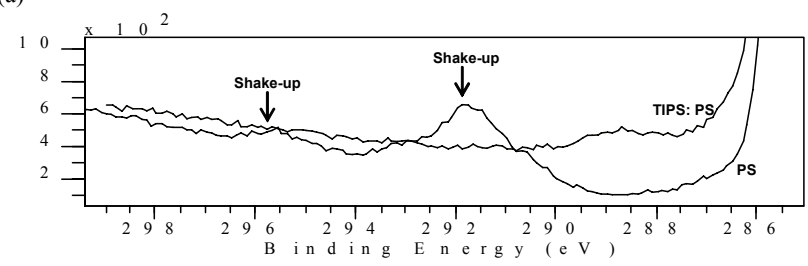

(b)

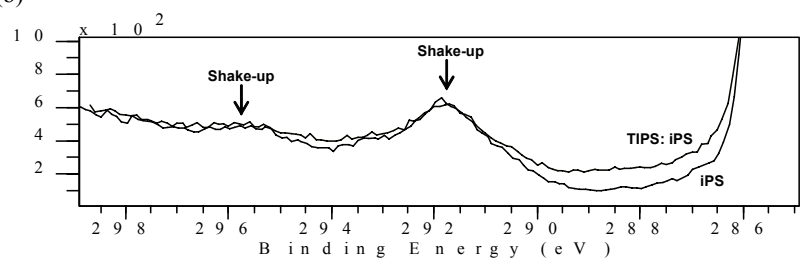

FIG. 3: Comparison of high resolution XPS C $1 s$ spectra for insulting binder and TIPS-pentacene:binder (50:50) for (a) PS and (b) iPS.

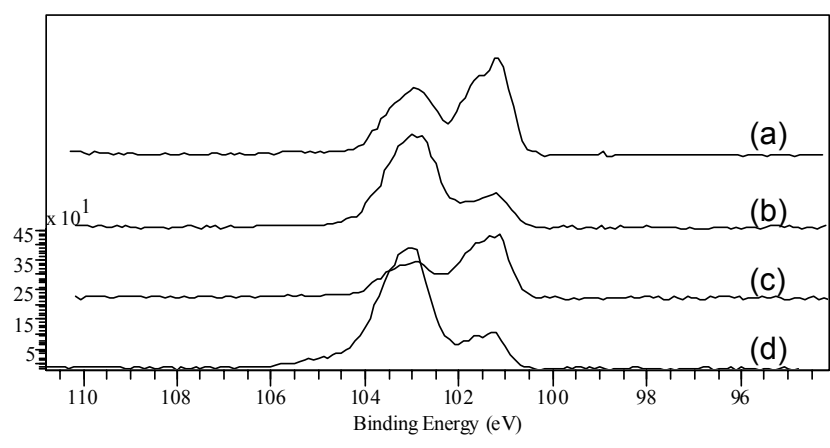

FIG. 4: High resolution spectra of $\mathrm{Si}(2 p)$ for TIPSPentacene:iPS for the following conditions: (a) 10:90 active interface, (b) 10:90 top interface, (c) 50:50 active interface and (d) 50:50 top interface.

is found to reduce in all binders, with the lowest concentration of TIPS-pentacene in semi-crystalline binder showing a characteristic which is virtually hysteresis-free.

Figure 2 shows the evolution of crystalline structure of TIPS-Pentacene: iPS with TIPS-Pentacene fraction. For high concentration of TIPS-Pentacene the crystalline phase organises as platelets like the pure material. Phase segregation with the binder is noticeable as iPS appears wrapped around the crystal features. For lower concentration of TIPS-pentacene, we do not observe the same phase segregation between the binder and the crystalline semiconductor, but instead of organizing like platelets, crystals are more dendritic. We notice that for low TIPSpentacene fraction, the crystal is highly branched indicative of slow growth. The privileged direction of the crystal is parallel to the $(1 \overline{2} 0)$ plane and the acene molecules tend to align along the (120). It has been observed that upon heating [6], defects appearing the crystal cause void that are parallel to (210) and then (120). Fractures appear along the (120) due to cleavage and slipping of molecules along the acene planes. Mechanical hindrance to crystal growth due to intercalation of the binder should then cause defect along these two privileged $(h k l)$ planes.

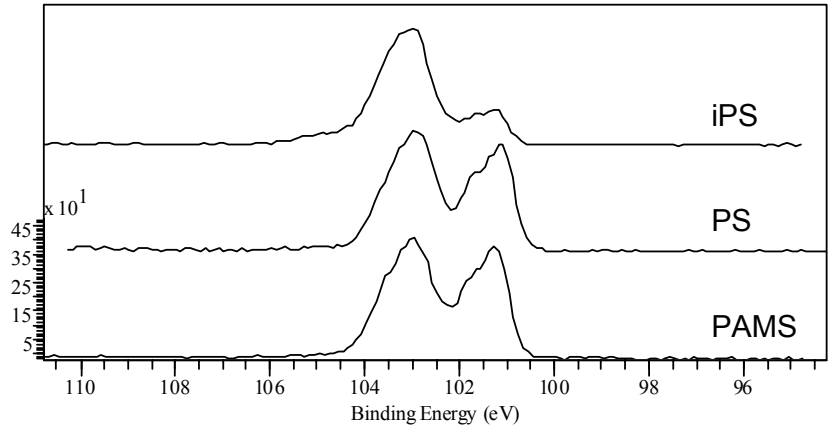

FIG. 5: High resolution spectra of $\mathrm{Si}(2 p)$ for TIPSPentacene:binder 50:50 for the top interface.

TABLE II: Quantification of Silicon over carbon signal at the top interface in three formulated TIPS-pentacene:binder when fraction of TIPS-pentacene:binder is 50:50. Quantification were estimated from wide XPS scan of the various samples

\begin{tabular}{ccc}
\hline \hline Binder & Carbon $\%$ & Silicon $\%$ \\
\hline PAMS & 91.2 & 8.8 \\
PS & 91.1 & 8.9 \\
iPS & 93.9 & 6.1 \\
\hline \hline
\end{tabular}

Thanks to this segregation the crystalline structure is preserved at such dilution. For comparison Fig. 2(c) shows the amorphous nature of TIPS-Pentacene:PS 10:90. At such dilution the crystalline structure of the organic semiconductor is completely disrupted giving rise to crystalline islands of TIPS-pentacene dispersed in amorphous binder, consistent with very low saturated hole mobility.

In the XPS data, the presence of shake-up satellites on the high binding energy side of the main core level corresponds to the competitive phenomenon of the ejection of a photoelectron and promotion of an occupied-unoccupied $\left(\pi-\pi^{*}\right)$ transition within an isolated benzene ring or other localised conjugated part of the molecule [7]. We focused our observation on the high resolution $\mathrm{C} 1 s$ spectra in the shake-up region as shown in Fig. 3. The area of the shakeup satellite which can reach up to $10 \%$ of the general C $1 s$ peak and for PAMS, PS, iPS and iPVBP is observed at binding energies of $291.5 \mathrm{eV}$ and $295.5 \mathrm{eV}$. The intensity of the shake-up satellite for the binder is independent of whether it is amorphous or crystalline. When blended with TIPS-pentacene the carbon shake-up satellite of the amorphous binder disappears indicating that the conjugation length has increased significantly. We ascribe this to evidence of TIPS-pentacene-binder interaction within the solid film. When blended with TIPS-pentacene the carbon shake-up satellite of the crystalline binder is not affected indicating no interaction between the two.

Figure 4 focuses on the high resolution $\operatorname{Si}(2 p)$ for the formulation TIPs-pentacene:iPS for the highest and the lowest concentration in TIPS-pentacene. Comparison with the XPS data simply for an OTS self assembly monolayer (SAM) treated wafer confirms that the dual signal observed for $\mathrm{Si}(2 p)$ for blends is not due to either the OTS or wafer silicon being observed through the thin organic 
thin film. Literature has already extensively studied the signature of $\mathrm{Si}(2 p)$ of silica wafer and silane $\mathrm{SAM}$ treatment [8-10] and corroborates this observation. We observe that the silicon peak is a doublet, with the peak at the highest binding energy corresponding to silicon linked to a hydrogen rich carbon environment. This is either due to the triisopropyl silyl environment or maybe to some week intermolecular interaction with the neighbour backbone of isotactic polystyrene. We notice another peak in the vicinity of $100 \mathrm{eV}$. We rule out the possibility of observing the $\operatorname{Si}(2 p)$ (means Silicon metal) because of the thickness of the organic film and the silica layer. Li et al. [11] have observed that this peak can be attributed to silicon bonded to a carbon with a poor hydrogen environment, which could correspond in the TIPS-pentacene to the ethynyl linked to the silicon atom. In both formulations, the relative percentage of $\mathrm{SiC}$ is greater at the bottom interface than at the top interface, which could demonstrate a higher concentration of TIPS-pentacene at the active interface of the OFET. Figure 5 shows the high resolution $\operatorname{Si}(2 p)$ for TIPS-pentance:binder (50:50) top interface. The crystalline binder is the only one exhibiting a difference between the two signals. Quantifications of the peaks are compiled in Table II. The calculation is relative to the area of the peak and does not take into account any RSF difference considering that these two sub-regions are within the same elemental peak. Quantification of the silicon relative to carbon for various binders validates the fact that the top surface of TIPS-Pentacene:iPS is depleted in silicon with respect to that calculated for a simple blend. This confirms that when formulated with a crystalline binder, stratification of the film forces TIPSPentacene towards the active interface which is no more than a few atomic layers thick [12], thus preserving the hole mobility.

\section{CONCLUSIONS}

The electronic performance of TIPS-pentaceneinsulator binder blends is highly dependent upon the crystalline morphology of TIPS-pentacene within the film. The development of morphology within the film is the result of a "double-percolation"-like mechanism where liquid-liquid phase separation at the early stages of drying is followed by segregation within the solidifying film caused by crystallization of one or both of the components.

In the case of amorphous binder there is evidence for strong interaction between TIPS-pentacene and binder in the solidified film leading to a simple blend. We see no evidence of segregation towards the active interface. When amorphous high molecular weight PS is used, the driving force for liquid-liquid phase separation is greater but again there no evidence of phase separation. Since there is no stratification towards the active interface saturated hole mobility falls away rapidly at weight fractions of TIPSpentacene $<0.5$, becoming inexistent when the film is completely amorphous.

In the case of high molecular weight semi-crystalline binder iPS, we observe phase segregation even down to low weight fraction of active material. We believe this stratification is a consequence of TIPS-pentacene crystallizing first from solution with segregation being driven by enthalpic interactions arising from subsequent binder crystallization. XPS analysis confirms that TIPSPentacene is more concentrated towards the active interface no matter its dilution within the crystalline binder. As a consequence the saturated hole mobility remains almost independent of TIPS-pentacene dilution.

\section{Acknowledgments}

The authors would like to thank the UK Home Office for funding.
[1] O. D. Jurchescu, J. Baas, and T. M. Palstra, Appl. Phys. Lett. 84, 3061 (2004).

[2] J. E. Anthony, J. S. Brooks, D. l. Eaton, and S. R. Parkin, J. Am. Chem. Soc. 123, 9482 (2001).

[3] D. Feili, M. Schuettler, T. Doerge, S. Kammer, and T. Stieglitz, Sens. Actuator A 120, 101 (2005).

[4] Torres and D. M. Taylor, J. Appl. Phys. 98, 073710 (2005).

[5] A. R. Brown, C. P. Jarrett, D. M. de Leeuw, and M. Matters, Synthetic metals 88, 37 (1997).

[6] J. Chen, C. K. Tee, J. Yang, C. Shaw, M. Shtein, J. Anthony, and D. Martin, J. Polym. Sci. B. Polym. Phys. 44, $3631(2006)$.
[7] D. Briggs, Surface analysis of polymers by XPS and static SIMS (Cambridge University Press, Cambridge, 2005).

[8] Y. C. Araujo, P. G. Toledo, V. Leon, and H. Y. Gonzalez, J. Coll. Inter. Sci. 176, 485 (1995).

[9] J. Dong, A. Wang, K. Y. Simon, and G. Mao, Thin Solid Films 515, 2116 (2006).

[10] P. Jiang, S-Y. Li, H. Sugimura, and O. Takai, Appl. Surf. Sci. 252, 4230 (2006).

[11] Li, Z. Cui, and M. A. Baker, Surf. Interface Anal. 36, 1254 (2004).

[12] D. M. Goldie, T. J. Dines, J. Phys. D: Appl. Phys. 40, 982 (2007). 